\title{
APPLICATION OF LAPLACE TRANSFORM HOMOTOPY PERTURBATION METHOD TO GAS DYNAMIC EQUATION: A MODIFIED APPROACH
}

\author{
Prem Kiran G. Bhadane ${ }^{1}$, V. H. Pradhan ${ }^{2}$ \\ ${ }^{1}$ Assistant Professor, Department of Applied Sciences, RCPIT, Maharashtra, India, \\ om.bhadane@rediffmail.com \\ ${ }^{2}$ Associate Professor, Department of Applied Mathematics And Humanities, SVNIT, Gujarat, India \\ pradhan65@yahoo.com
}

\begin{abstract}
The Laplace transform homotopy perturbation method (LTHPM) is previously used to solve gas dynamic equation. However, in the present paper a modified approach of Laplace transform homotopy perturbation method is used to obtain the solution of gas dynamic equation. The present modification detects the solution with less numerical computational work as compared to the earlier approach. Also, in this modification no restrictive assumptions are considered and therefore reduces the numerical computations to a greater extent. The obtained solutions are compared with the available exact solutions and LTHPM solution. It shows that the modified approach of LTHPM has a good agreement with the exact solution and LTHPM.
\end{abstract}

Keywords: Laplace Transform, Gas Dynamic Equation and Laplace Transform with Homotopy Perturbation Method.

\section{INTRODUCTION}

LTHPM is the method in which the Laplace transform (LT) is coupled with HPM i.e. homotopy perturbation method for the approximate analytical solution of nonlinear differential equations. This combination is easy, efficient and widely useful for solving nonlinear differential equations. In HPM, a homotopy is constructed by introducing an embedding parameter $p \in[0,1]$. The HPM uses the small parameter $p$ and the solution is written as a power series. The changing process from zero to unity is called deformation in Topology.[5] The nonlinear term can be decomposed as a power series in $p$ in terms of He's polynomials which can be generated by several means.

Mostly various physical phenomena are governed by linear or nonlinear differential equations. Although several techniques like Laplace transform, Fourier transform and Sumudu transform are available for solving the linear differential equations, the solution of nonlinear differential equation can be obtained by combining these techniques with other analytical methods to handle the nonlinearities. Recently, many researchers have combined Laplace transform with HPM, ADM and VIM.

The gas dynamics nonlinear partial differential equation as given below [3]

$\frac{\partial u}{\partial t}+u \frac{\partial u}{\partial x}-u(1-u)=\varnothing(x, t), 0 \leq x \leq 1, t \geq 0$

Gas dynamics is one of the branch of fluid dynamics. The mathematical expression of gas dynamics equations is built on the physical laws of conservation. The nonlinear gas dynamic equations in fluid dynamics have been solved by Jafari et al. (2008) [2], Evans and Bulut (2002) [1], Hossein et al. (2008) [4] by applying various kinds of analytical methods such as HPM, decomposition method and variational iteration method. Hossein Aminikhah et al. (2013) [3] has applied the LTHPM to get the solution of nonlinear gas dynamic equation. However, in the present paper the modified LTHPM [6, 8 and 9] is applied to solve nonlinear gas dynamic equation. The proposed alteration will speed up the quick convergence of series solution when compared with LTHPM and hence delivers major improvement. In the next section, equation (1) is solved by using modified LTHPM for the two cases $\emptyset(x, t)=0$ and $\emptyset(x, t) \neq 0$ with specific initial condition to show the effectiveness and the usefulness of modified LTHPM.

\section{APPROXIMATE ANALYTICAL SOLUTION OF GAS DYNAMIC EQUATION}

In the present section the approximate analytical solution of equation (1) is obtained for each of the cases $\emptyset(x, t)=$ 0 and $\varnothing(x, t) \neq 0$.

2.1 For $\emptyset(x, t)=0$ equation (1) can be written as follow

$\frac{\partial u}{\partial t}+u \frac{\partial u}{\partial x}-u(1-u)=0,0 \leq x \leq 1, t \geq 0$

with the condition

$u(x, 0)=a e^{-x}$

For equation (2) exact solution is [7]

$u(x, t)=a e^{t-x}$ 
Take LT on both sides of equation (2) and using properties of $\mathrm{LT}$, equation (2) reduce to

$L[u(x, t)]=\frac{u(x, 0)}{s}+\frac{1}{s} L\left[u-u^{2}-u u_{x}\right]$

Using initial condition (3) and inverse LT, equation (5) reduce to

$u(x, t)=a e^{-x}+L^{-1}\left\{\frac{1}{s} L\left[u-u^{2}-u u_{x}\right]\right\}$

Invoking the homotopy perturbation method,

$\sum_{n=0}^{\infty} p^{n} u_{n}(x, t)=a e^{-x}$

$+p L^{-1}\left\{\frac{1}{s} L\left[\sum_{n=0}^{\infty} p^{n} u_{n}(x, t)-\sum_{n=0}^{\infty} p^{n} H_{n}(u)\right]\right\}$

Here,He's polynomials are denoted by $H_{n}(u)$. The first three components are

$H_{0}(u)=u_{0}^{2}+u_{0} \frac{\partial u_{0}}{\partial x}$

$H_{1}(u)=2 u_{0} u_{1}+u_{0} \frac{\partial u_{1}}{\partial x}+u_{1} \frac{\partial u_{0}}{\partial x}$,

$H_{2}(u)=2 u_{0} u_{2}+u_{0} \frac{\partial u_{2}}{\partial x}+u_{1} \frac{\partial u_{1}}{\partial x}+u_{2} \frac{\partial u_{0}}{\partial x}+u_{1}^{2}$,

and so on.

Equate the coefficient of power of $p$ in equation (7),

$p^{0}: u_{0}(x, t)=a e^{-x}$

$$
\begin{aligned}
p^{1}: u_{1}(x, t) & =L^{-1}\left\{\frac{1}{s} L\left[u_{0}-H_{0}(u)\right]\right\} \\
& =L^{-1}\left\{\frac{1}{s} L\left[u_{0}-u_{0}^{2}-u_{0} \frac{\partial u_{0}}{\partial x}\right]\right\}=a e^{-x} t \\
p^{2}: u_{2}(x, t) & =L^{-1}\left\{\frac{1}{s} L\left[u_{1}-H_{1}(u)\right]\right\} \\
& =L^{-1}\left\{\frac{1}{s} L\left[u_{1}-2 u_{0} u_{1}-u_{0} \frac{\partial u_{1}}{\partial x}-u_{1} \frac{\partial u_{0}}{\partial x}\right]\right\} \\
& =L^{-1}\left\{\frac{1}{s} L\left[a e^{-x} t\right]\right\}=a e^{-x} \frac{t^{2}}{2 !}, \\
p^{3}: u_{3}(x, t) & =L^{-1}\left\{\frac{1}{s} L\left[u_{2}-H_{2}(u)\right]\right\} \\
& =L^{-1}\left\{\frac { 1 } { s } L \left[u_{2}-2 u_{0} u_{2}-u_{0} \frac{\partial u_{2}}{\partial x}-u_{1} \frac{\partial u_{1}}{\partial x}\right.\right. \\
& \left.\left.-u_{2} \frac{\partial u_{0}}{\partial x}-u_{1}^{2}\right]\right\}=a e^{-x} \frac{t^{3}}{3 !},
\end{aligned}
$$

In this way, the further approximations can be obtain,

and so on.

$$
\begin{aligned}
& p^{4}: u_{4}(x, t)=a e^{-x} \frac{t^{4}}{4 !} \\
& p^{5}: u_{5}(x, t)=a e^{-x} \frac{t^{5}}{5 !}
\end{aligned}
$$

Substituting above values in the following equation

$$
\begin{aligned}
& u(x, t)=u_{0}(x, t)+u_{1}(x, t)+u_{2}(x, t)+\ldots \\
& u(x, t)=a\left(e^{-x}+e^{-x} t+e^{-x} \frac{t^{2}}{2 !}+e^{-x} \frac{t^{3}}{3 !}+e^{-x} \frac{t^{4}}{4 !}+\cdots\right) \\
& u(x, t)=a e^{-x}\left(1+t+\frac{t^{2}}{2 !}+\frac{t^{3}}{3 !}+\frac{t^{4}}{4 !}+\frac{t^{5}}{5 !}+\cdots\right) \\
& u(x, t)=a e^{t-x}
\end{aligned}
$$

This is the solution of equation (2) with condition (3) and same as the exact solution and LTHPM solution.Fig. 1 shows the graph ofu $=\mathrm{e}^{\mathrm{t}-\mathrm{x}}, \mathrm{t}>0$ and $0 \leq x \leq 1$.

2.2 For $\emptyset(x, t) \neq 0$ and $\emptyset(x, t)=-e^{t-x}$ equation (1) can be written as follow

$\frac{\partial u}{\partial t}+u \frac{\partial u}{\partial x}-u(1-u)=-e^{t-x}$

with the condition

$u(x, 0)=1-e^{-x}$

For equation (9) exact solution is [7]

$u(x, t)=1-e^{t-x}$

Take LT on both sides of equation (9) and using properties of LT, equation (9) reduce to

$$
\begin{gathered}
L\left[\frac{\partial u}{\partial t}\right]=L\left[u-u^{2}-u \frac{\partial u}{\partial x}\right]-L\left[e^{t-x}\right] \\
L[u(x, t)]=\frac{u(x, 0)}{s}+\frac{1}{s} L\left[u-u^{2}-u u_{x}\right] \\
-e^{-x} \frac{1}{s(s-1)}
\end{gathered}
$$

Using initial condition (10) and inverse Laplace transform equation (13) reduce to

$$
\begin{aligned}
& u(x, t)=\left(1-e^{-x}\right) \\
& +L^{-1}\left\{\frac{1}{s} L\left[u-u^{2}-u u_{x}\right]-\frac{1}{s} e^{-x} \frac{1}{s-1}\right\}
\end{aligned}
$$

Since

$L^{-1}\left\{e^{-x} \frac{1}{s(s-1)}\right\}=e^{-x}\left(-1+e^{t}\right)$

Using equation (15), equation (14) can be written as $u(x, t)=\left(1-e^{-x}\right)$

$+L^{-1}\left\{\frac{1}{s} L\left[u-u^{2}-u \frac{\partial u}{\partial x}\right]\right\}+e^{-x}-e^{t-x}$

Invoking the homotopy perturbation method,

$\sum_{n=0}^{\infty} p^{n} u_{n}(x, t)=\left(1-e^{t-x}\right)$
$+p L^{-1}\left\{\frac{1}{s} L\left[\sum_{n=0}^{\infty} p^{n} u_{n}(x, t)-\sum_{n=0}^{\infty} p^{n} H_{n}(u)\right]\right\}$

HereHe's polynomials are denoted by $H_{n}(u)$. The first three components are

$H_{0}(u)=u_{0}^{2}+u_{0} \frac{\partial u_{0}}{\partial x}$,

$H_{1}(u)=2 u_{0} u_{1}+u_{0} \frac{\partial u_{1}}{\partial x}+u_{1} \frac{\partial u_{0}}{\partial x}$,

$H_{2}(u)=2 u_{0} u_{2}+u_{0} \frac{\partial u_{2}}{\partial x}+u_{1} \frac{\partial u_{1}}{\partial x}+u_{2} \frac{\partial u_{0}}{\partial x}+u_{1}^{2}$,

and so on.

Equate the coefficient of power of $p$ in equation (17),

$p^{0}: u_{0}(x, t)=1-e^{t-x}$, 


$$
\begin{aligned}
p^{1}: u_{1}(x, t)= & L^{-1}\left\{\frac{1}{s} L\left[u_{0}-H_{0}(u)\right]\right\} \\
= & L^{-1}\left\{\frac { 1 } { s } L \left[\left(1-e^{t-x}\right)-\left(1-e^{t-x}\right)^{2}\right.\right. \\
& \left.\left.-\left(1-e^{t-x}\right) e^{t-x}\right]\right\} \\
= & L^{-1}\left\{\frac{1}{s} L[0]\right\}=0
\end{aligned}
$$

In this way, the further approximations can be obtain,

$p^{2}: u_{2}=0$,

$p^{3}: u_{3}=0, \ldots$

Substituting above values in equation

$u(x, t)=u_{0}(x, t)+u_{1}(x, t)+u_{2}(x, t)+\ldots$

$u(x, t)=\left(1-e^{t-x}\right)+0+0+0 \ldots=1-e^{t-x}$

which is the exact solution of equation (9) with condition (10) and same as the exact solution and LTHPM solution. Fig. 2 shows the graph of $\mathrm{u}=1-\mathrm{e}^{\mathrm{t}-\mathrm{x}}, \mathrm{t}>0$ and $0 \leq x \leq$ 1.

\section{CONCLUSIONS}

In the present paper a modified approach of LTHPM is shown for two nonlinear gas dynamic equations and compared with available exact solution and LTHPM solution [3]. On comparing the obtained solution by a modified approach of LTHPM with the exact solution and LTHPM solution, it is found that it matches well with the exact solution and LTHPM solution in each case. The present modification in LTHPM is reliable, elegant and yields the solution in a rapidly convergent sequence as compared to LTHPM. This proves that the present modification in LTHPM is a powerful mathematical method for solving non-linear gas dynamic equations and very effective and quite accurate. Hence, modification in LTHPM can be conveniently used in solving nonlinear partial differential equations arising in different fields of sciences.

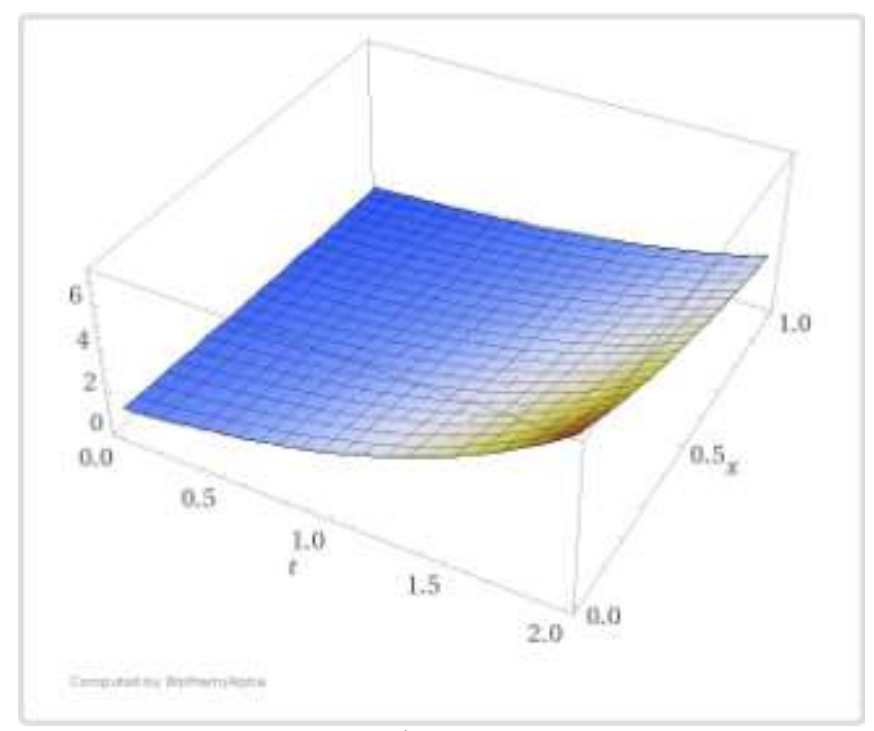

Fig.1: Graph of $\mathrm{u}=\mathrm{e}^{\mathrm{t}-\mathrm{x}}, \mathrm{t}>0$ and $0 \leq x \leq 1$.

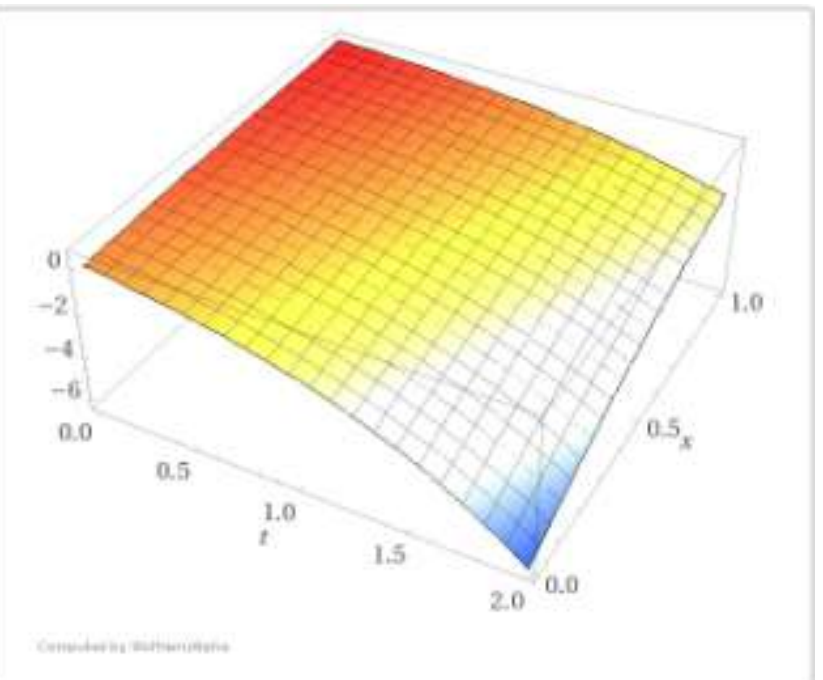

Fig.2: Graph of $\mathrm{u}=1-\mathrm{e}^{\mathrm{t}-\mathrm{x}}, \mathrm{t}>0$ and $0 \leq x \leq 1$.

\section{REFERENCES}

[1]. D.J. Evans, H. Bulut (2002), A new approach to the gas dynamics equation: An application of the decomposition method, Appl. Comp. Math., 79, 817822.

[2]. H. Jafari, M. Zabihi, M. Saidy (2008), Application of homotopy perturbation method for solving gas dynamics equation, Appl. Math. Scie.,2, 2393-2396.

[3]. Hossein Aminikhah, Ali Jamalian (2013), Research Article: Numerical Approximation for Nonlinear Gas Dynamic Equation, International Journal of Partial Differential Equations, Vol.2013, Article ID 846749, 7 pages.

[4]. HosseinJafari, Hassan Hosseinzadehb and ElhamSalehpoor (2008), A New Approach to the Gas Dynamics Equation: An Application of the Variational Iteration Method, Applied Mathematical Sciences, 2(48), 2397-2400.

[5]. Hradyesh Kumar Mishra, Atulya K. Nagar (2012), HeLaplace Method for Linear and Nonlinear Partial Differential Equations, Journal of Applied Mathematics, Hindawi Publishing Corporation, Volume 2012, Article ID 180315, 16 pages.

[6]. Jagdev Singh, Devendra Kumar and Sushila (2012), homotopy perturbation algorithm using laplace transform for gas dynamics equation, Journal of the Applied Mathematics, Statistics and Informatics (JAMSI), 8(1), 55-61.

[7]. M. Matinfar, M.Saeidy, M. Mahdavi and M. Rezael (2011), Variational iteration method for exact solution of gas dynamics equation using He's polynomials, Bulletin of Mathematical Analysis and Applications, 3(3), 50-55.

[8]. Sumit Gupta, Devendra Kumar and Jagdev Singh (2015), Analytical solutions of convection-diffusion problems by combining Laplace transform method and homotopy perturbation method, Alexandria Engineering Journal, 54, 645-651.

[9]. Yasir Khan, Qingbiao Wu (2011), Homotopy perturbation transform method for nonlinear equations using He's polynomials, Computers and Mathematics with Applications, 61, 1963-1967. 